\title{
Horizons/Théâtre
}

Revue d'études théâtrales

8-9| 2016

Théâtres du geste, du jeu et de la voix

\section{Les plis du jeu : référentialité, résillusion et ekphrasis dans Le Goût du faux et autres chansons}

\section{Emmanuel Cohen}

\section{(2) OpenEdition}

1 Journals

Édition électronique

URL : https://journals.openedition.org/ht/809

DOI : $10.4000 /$ ht.809

ISSN : 2678-5420

Éditeur

Presses universitaires de Bordeaux

Édition imprimée

Date de publication : 31 décembre 2016

Pagination : 84-96

ISSN : 2261-4591

\section{Référence électronique}

Emmanuel Cohen, «Les plis du jeu : référentialité, résillusion et ekphrasis dans Le Goût du faux et autres chansons ", Horizons/Théâtre [En ligne], 8-9 | 2016, mis en ligne le 01 janvier 2018, consulté le 22 mai 2022. URL : http://journals.openedition.org/ht/809 ; DOI : https://doi.org/10.4000/ht.809

\section{cc) (1) $\odot$}

La revue Horizons/Théâtre est mise à disposition selon les termes de la Licence Creative Commons Attribution - Pas d'Utilisation Commerciale - Pas de Modification 4.0 International. 


\section{EMMANUEL COHEN}

Emmanuel Cohen est docteur en Arts de la scène. Sa thèse, soutenue en 2014 et intitulée Le Théâtre Nondramatique, traite de la critique de la forme dramatique chez Gertrude Stein, Dada et le surréalisme. Il a publié des articles sur les théâtres des avant-gardes historiques dans diverses revues dont Théâtre/Public, Agôn et prochainement dans L'Annuaire théâtral. En septembre 2013, il a organisé avec Christophe Bident (Professeur à l'Université de Picardie Jules Verne) et Laure Couillaud un colloque international intitulé « Le théâtre pense, certes... Mais quoi ? Comment? Et où ? », dont les actes ont paru dans la revue Incertains Regards (PUP) en mai 2015. Depuis septembre 2014, il enseigne l'histoire des arts et le français à TNS Parsons Paris. II est aussi auteur multimédia pour la Bibliothèque nationale de France.

Mail : cg.emmanuel@gmail.com

Résumé: Cet article analyse le fonctionnement de certains principes ludiques, comme la résillusion, dans Le Goût du faux et autres chansons de Jeanne Candel et du collectif La Vie Brève. En questionnant les procédés de l'écriture théâtrale de Candel, nous verrons comment l'immersion du spectateur est encouragée sur un double plan : d'abord cognitif, par la nature des images théâtrales et le jeu des références, et ensuite affectif, par la création d'un espace de résillusion sur scène. Une analyse détaillée du fonctionnement de l'ekphrasis théâtrale nous permettra ensuite de spécifier la nature de l'expérience

Abstract: This article aims to analyze the way certain ludic principles, as "résillusion", in Le Goût du faux et autres chansons by Jeanne Candel and the artistic collective La Vie Brève. By questioning the different processes of Candel's stage writing, we will see how the viewer's immersion is encouraged on two levels: first on a cognitive one, by the nature of the images on the stage and the play with references, and then on an affective one, through the creation of a space of "résillusion" on stage. A detailed analysis of the way the dramatic ekphrasis works will allow us to specify the nature of the theat- théâtrale ainsi créée. Si dans un premier temps les principes ludiques favorisent la connaissance pathétique à la connaissance intellectuelle ou critique, leur fonctionnement à l'intérieur d'un dispositif théâtral autoréflexif caractérisé par la forme-sens du pli baroque permet néanmoins de formuler certaines interprétations quant au sens de ce spectacle dans notre société contemporaine.

Mots-clés: Jeanne Candel, théâtre, ludisme, ekphrasis, résillusion

rical experience as it is. If, at first, the ludic principles favor a pathetic knowledge over an intellectual or critical knowledge, the way they function inside of an auto-reflexive theatrical apparatus, characterized by the meaning-form of the baroque fold, allows nonetheless to express some interpretations as for the meaning of the show in our modern society.

KeYwords: Jeanne Candel, theater, ludic principles, ekphrasis, résillusion 


\section{Les plis du jeu : référentialité, résillusion et ekphrasis dans Le Goût du faux et autres chansons}

\section{Situation}

Le Goût du faux et autres chansons, création de 2014 de Jeanne Candel et du collectif La Vie Brève, est une pièce à la composition labyrinthique. Les références à des œuvres picturales plus souvent qu'à des pièces de théâtre et les saynètes nées d'improvisations forment les motifs chatoyants et jouissifs d'une trame dramatique pour le moins lâche, car hétérogène, voire totalement éclatée.

Cette fois-ci j'ai utilisé deux méthodes. La première est de travailler à partir de matériaux : les Métamorphoses, la Genèse, les textes d'Aby Warburg (m'intéresse surtout son idée qu'il y a des images qui persistent, des survivances du passé dans le présent) mais aussi des textes scientifiques sur la formation de l'univers ou le renouvellement des cellules ${ }^{1}$.

En ce sens, Le Goût du faux fait figure de parangon de ces créations contemporaines fragmentées qui, par cette profusion de matériaux convoqués sur le plateau, sont de véritables casse-têtes formels, des sortes d'hydres postdramatiques $^{2}$. Cette hétérogénéité de forme et de contenu invite à repenser l'objet même de la représentation théâtrale, qui réside alors a priori autant dans le fait de faire - la performance - que dans celui de montrer - ce qui est représenté (au sens de mimèsis ${ }^{3}$ ).

Le titre, Le Goût du faux et autres chansons, inscrit de même le spectacle dans un certain héritage esthétique, celui de la facticité ou de l'artificialité du théâtre, et invite à s'interroger sur ce qu'il peut réaliser. Suivant cette hypothèse, le faux, la nature factice du spectacle, serviraient d'élément fédérateur, de force centripète maintenant ensemble des saynètes et micro-performances a priori autonomes les unes des autres. La revendication de cette non-conformité au naturel et à la vérité 4 servirait alors de principe compositionnel de la représentation, au détriment d'une fable unifiante elle-même davantage liée au textes, et donc peu pertinente dans le cas du théâtre de plateau. Ce titre sonne ainsi comme un écho à l'histoire de la réception 
du théâtre et à ses limites. Le « faux » rappelle l'opposition, maintes fois combattue, entre le plaisir né de la représentation et l'entorse morale que ce rassemblement génèrerait. Replacé dans le contexte des nouvelles créations contemporaines ${ }^{6}$, il semble indiquer à la foisl'intériorisation et le dépassement de cette condamnation morale du théâtre - cette antienne philosophique. Il annonce un dispositif théâtral moins destiné à créer un effet de réel ou de vraisemblable, qu'un jeu sur les conditions de réceptions de ses spectateurs?. Ce qui se joue dans Le Goût du faux serait alors la capacité pour l'acteur de s'emparer du plateau et du théâtre dans sa « phénoménalité, l’apparaitre de son être-là ${ }^{8} \gg$.

Nous verrons, dans cet article, comment la mise en scène joue constamment de l'image théâtrale comme espace ludique et heuristique grâce à des procédés tels que l'hyper-référentialité des images et l'ekphrasis qui ménagent des plis ${ }^{9}$ et des replis dans la trame d'une représentation baroquisante ${ }^{10}$.

\section{Un vertige ludique des images}

\section{Une scène vertigineuse}

Dès les premières minutes du spectacle, l'absence sensible de fable produit un basculement d'intérêt chez les spectateurs : l'attention se tourne sur ce que chaque scène réalise, par les dialogues et les images. Il convient donc de commencer par décrire ce que le spectateur voit.

La représentation s'ouvre sur l'entrée d'une femme en robe de soirée. Elle traverse la scène de jardin à cour alors que son apparition impose le silence dans la salle. Cette image évoque l'esthétique de Pina Bausch par le costume (une robe de soirée), la gestuelle précise et l'intrusion d'un objet du quotidien incongru sur le plateau épuré. Une fois installée au piano, l'image « déraille », comme c'est parfois le cas aussi chez Bausch : elle n'actionne pas la pédale du piano, mais celle d'une machine à coudre accrochée à sa traine. Entraînée, littéralement, à l'extérieur de la scène par la force mécanique de la machine qui avale le tissu, elle disparaît en coulisse sans qu'un mot n'ait été prononcé.

Une deuxième image prend alors forme autour de ce piano. Une chanteuse, un pianiste et un violoncelliste vaguement habillés comme des Flamands du $\mathrm{XVII}^{\mathrm{e}}$ siècle incarnent les personnages de cette scène de genre. Ils sont accompagnés d'un « homme mystérieux », à moitié caché derrière le rideau de scène. Il s'agit d'une Leçon de musique, prétendument inspirée par un peintre flamand du même siècle, dont le nom et l'identité précise se perdent dans les explications contradictoires des personnages qui jouent et commentent simultanément la scène. La tentative d'exégèse par des acteurs-personnages, qui ne 
cessent de sortir de leur rôle, prend bientôt le pas sur la leçon de musique ellemême et débouche sur une ekphrasis théâtrale. La scène de genre se résorbe en chorale cacophonique : le dénouement vient non pas de l'interprétation de l'argument de la scène de genre, mais de son exégèse poussée jusqu'à la surinterprétation et à l'éclatement de la proposition. De la narration d'une fiction, qui serait le propre d'un tableau italien, l'on glisse vers une scrutation minutieuse des détails, un mode d'exégèse plus fidèle à l'esthétique de cette peinture flamande ${ }^{11}$. Ainsi, la leçon de musique se transforme-t-elle en une leçon de dissection d'insecte qui évoque une version loufoque de La Leçon d'anatomie du docteur Tulp de Rembrandt.

À ce glissement thématique succède un autre, spatio-temporel celui-là : $\mathrm{du}$ fond de scène, un couple en habits contemporains débarque, des sacs de randonnée sur le dos. Ils se pressent pour retrouver Michel, un écrivain dépressif, installé en avant-scène, qui les a invités à dîner. L'action se resserre autour de ces trois personnages installés à une table dressée pour l'occasion, devant un imposant îlot de cuisine installé à jardin. Une structure en bois délimite, au-dessus de leurs têtes, cette cuisine équipée, et apporte un effet de réel absent de la pièce jusqu'à présent. Les accents geignards et cyniques de Michel, à la manière d'un Jean-Pierre Bacri, comme lui dégarni et tout de noir vêtu, complètent ce qui ressemble à une référence à un certain type de théâtre de genre, comme Cuisine et dépendances, ou de cinéma, français à l'instar du Goût des autres ${ }^{12}$, une citation qui transparaît dans le titre de la pièce même.

L'incommunicabilité entre les personnages est poussée jusqu'à son paroxysme, et les invités de Michel quittent bientôt la scène, ce qui, d'une certaine manière, provoque l'éclatement de la situation dramatique. Face à cette apparente impasse dramatique, le jeu est relancé par une apparition invraisemblable, au sens où elle ne respecte pas les règles du théâtre dramatique. Une femme de petite taille, en robe bustier, surgit sur le plateau et se plante devant Michel, le romancier en panne d'inspiration. Dans une frénésie qui relève moins du jeu réaliste que du jeu de rapidité, elle enchaîne les répliques décousues dans lesquelles apparaissent des références au Décaméron de Boccace ou au mythe de Médée. À travers les réactions de l'écrivain, on comprend alors que la femme synthétise en elle un ensemble de personnages tirés de ses romans ou de sources d'inspiration. La scène prend une signification symbolique et métathéâtrale : à l'incapacité de dire et de raconter des histoires de Michel, est substitué le jeu de ce «personnage-palimpseste » fondé sur la performativité et le spectaculaire. C'est dans ce sens d'emballement du plateau que peuvent être comprises les images scéniques qui succèdent à cette scène, d'abord muettes puis de plus en plus bavardes, qui 
complètent cette première partie. Ainsi, voit-on successivement une femme se préparer comme l'on prépare un rôti, à force de persil dans les oreilles, de pomme dans la bouche et de ficelle enroulée autour de la tête, et qui finit la tête dans le four allumé. Une seconde propose aux spectateurs ses globes oculaires fraîchement arrachés dans un cornet de glace, après leur avoir offert deux boules de sorbet à la framboise, ce qui provoque le rire et l'effroi dans la salle. Enfin, deux astronautes donnent une interview en duplex avec la Terre depuis leur station spatiale. Ainsi, les saynètes se succèdent, se superposent et se télescopent sans lien apparent, mais en cultivant toujours une dimension métathéâtrale et ludique.

\section{La scène : un espace de résillusion par excellence}

La description de la première moitié de la représentation donne à voir le jeu incessant de transformations scéniques et de changements du registre de jeu. Or, ces changements ne sont motivés par aucune règle dramatique, qu'elle soit chronologique - car la représentation ne s'organise autour d'aucun fil dramatique linéaire - ou logique - il n'y a pas d'unité d'action. $\mathrm{Au}$ contraire, une analyse de la scénographie permet de révéler un principe esthétique ludique reposant sur la capacité des spectateurs à comprendre les signes théâtraux.

Dans cette esthétique vertigineuse aux accents baroques, la résillusion ${ }^{13}$ est en effet reine. Les différents éléments du décor ne sont pas conçus en fonction d'un point de fuite, comme c'est le cas dans le théâtre à l'italienne, ou d'un centre, dans le « palais à volonté » du théâtre classique, mais comme des éléments disparates laissés à la disposition pour le jeu. Si une bâche blanche translucide recouvre tout le praticable, et quelques panneaux de bois posés au fond sur le mur du théâtre arrêtent le regard au lointain, ces éléments ne sont pas suffisants pour créer un effet de perspective ou d'unité esthétique. La variété, au contraire, s'impose sur scène : un îlot de cuisine et une table à roulette réunis sous un faux toit en bois à jardin, un piano puis une table de pique-nique à cours, ainsi qu'un bac à roulette dont dépassent des branches de plantes exotiques et deux statues de lévriers à l'échelle, posées au lointain, complètent ce décor composite. En cela, il évoque davantage la bigarrure de la scène « ambulatoire » baroque et ses compartiments ${ }^{14}$. Les images scéniques ne prétendent pas imiter de façon illusionniste le réel, elles construisent des propositions spectaculaires imparfaites car visuellement non-illusionnistes ${ }^{15}$, mais théâtrales néanmoins.

De façon analogue, l'esthétique des costumes ménage une dimension métathéâtrale, dans la mesure où ces derniers ne sont ni des pièces histo- 
riques, ni des pièces ostentatoires, mais fonctionnelles ${ }^{16}$. Les comédiens ne portent pas de réelles combinaisons spatiales, mais de simples survêtements bleus dépareillés, l'un fait de tissu technique et l'autre en vulgaire molleton. Ce que le spectateur voit, ce n'est pas simplement un astronaute, mais un homme habillé en survêtement qui joue à l'astronaute : il se meut comme s'il était en apesanteur, ralentissant ses mouvements de façon grotesque ; il bruite avec la bouche le son des touches du tableau de bord, celui des portes d'un sas de pressurisation, et ainsi de suite. Dans ces scènes, l'acteur assume le caractère théâtral de ses actions. Ce faisant, il participe de ce que Lionel Abel

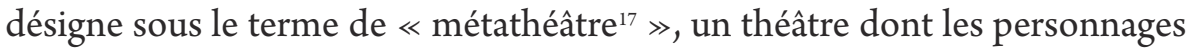
sont avant tout des créations théâtrales et conscientes de cette condition, pensées pour la scène et non des imitations ou des copies du monde réel. Chez Lionel Abel comme chez Jeanne Candel, la métathéâtralité va de pair avec un certain ludisme lié à l'enfance. Le survêtement, par sa forme souple et sa matière, évoque tout autant l'habit d'astronaute qu'il rappelle le jeu de l'enfant en pyjama, et fait écho à l'enromancement et au bovarysme ${ }^{18}$ contemporain auquel toute personne se prête. Sur la scène, le mensonge initial se transforme en illusion non trompeuse, mais « consentie » par le spectateur et productrice d'un plaisir intrinsèquement lié à la représentation théâtrale.

\section{Dans les plis de la représentation : le théâtre en scène}

Le jeu est ainsi traité comme le matériau même de la pièce : les scènes se télescopent, les acteurs changent de rôle et de registre de jeu, sans souci de réalisme ou de respect d'une illusion. À plusieurs niveaux, le théâtre sur le théâtre transparaît. Ce mode de composition dramaturgique joue sur « l'homomatérialité du signe théâtral ${ }^{19} \gg$, et en fait la matière même du jeu théâtral.

\section{L'homomatérialité du signe théâtral comme ressort du théâtral}

Jeanne Candel s'empare de cette dimension paradoxale du personnage : s'il est incarné par le corps de l'acteur - une matière -, il est aussi fiction. Le corps de l'acteur devient le lieu où se joue le sens de la pièce. L'homomatérialité est entièrement assumée : le fait que l'acteur soit un «matériau » ductile, et que cette dualité soit constitutive de la théâtralité, régit le fonctionnement ludique de la pièce, aussi bien que celle des scènes. Le peintre flamand et l'astronaute Kyril sont interprétés par le même acteur. Cette antinomie des rôles suscite surprise et plaisir à chaque nouvelle apparition de l'acteur. De même, l'actrice qui joue la sœur de l'écrivain apparaît seule en scène au milieu de la pièce. Différente de son rôle de sœur amoureuse et positive, l'actrice est habillée en robe de soirée, un costume qui fait à nouveau signe vers Pina 
Bausch et la scène d'ouverture. Arrivée en avant-scène, elle remplit bientôt des bocaux en verre de morceaux de son corps : un ongle dans l'un, un cheveu dans l'autre, une bouffée de cigarette dans un troisième. Cette scène rend sensible l'absence du personnage au profit d'un acteur-témoin, c'est-à-dire un acteur-signe qui n'incarne ou ne montre aucune fiction, mais qui crée de la théâtralité ${ }^{20}$ pure.

\section{De l'homomatérialité à l'optique dialectique}

La mise en scène pousse à son comble le principe d'homomatérialité du théâtre comme ressort de jeu. Les acteurs et les musiciens, les personnages et les techniciens se confondent allégrement sur scène.

Dans une autre scène, les astronautes entonnent un Motet de Schütz alors qu'ils déplient une antenne parabolique figurée par une simple table de pique-nique renversée. Cette image pauvre, par les moyens techniques mis en œuvre, n'en est pas moins complexe dans ses effets théâtraux. La table fonctionne comme un «trompe l'esprit ${ }^{21} \gg$ : à l'inverse du trompe-l'œil qui a pour but d'imiter à la perfection l'apparence d'une chose et qui doit se faire oublier, le trompe-l'esprit sélectionne certaines qualités d'un objet pour les recomposer sous une forme autre, qui requiert une interprétation pour faire sens. Il n'y a pas illusion, mais fabrication de signes. De même, si la musique jouée par les comédiens sert de bande-son cinématographique à l'action scénique $^{22}$, sa production de manière acoustique et visible en perturbe le fonctionnement conventionnel. En supprimant la dissociation physique entre la source d'émission de la musique et l'action scénique, le spectateur entend une « image de musique ${ }^{23}$ » ayant perdu sa qualité suggestive et sa capacité à créer une immersion affective. L'absence de médiation technique de la musique crée une distance comique et ludique par rapport à l'effet recherché. En d'autres termes, l'assemblage paradoxal de signes renvoyant à des imaginaires contradictoires crée une « optique dialectique ${ }^{24} \gg$.

L'optique dialectique ménagée dans les images scéniques subsume alors les questions d'intermédialité ou de rivalité entre les arts. Elle rethéâtralise chaque scène de la pièce. En effet, l'absence de costumes réalistes impose de trouver une autre façon de faire exister ces personnages. Les images sont théâtrales car elles résultent du jeu des acteurs qui simulent l'apesanteur par leurs gestes. Les bras et les jambes relevés au-dessus des épaules et du bassin signifient que les corps flottent en apesanteur bien que les acteurs soient assis sur des chaises qu'ils ont apportées avec eux, à vue des spectateurs. Cet effet métathéâtral est accentué par le traitement du son artificiellement désynchronisé : un décalage est simulé sur scène entre les voix et les corps lors de 
l'interview avec la Terre. La distance entre ces deux lieux ne pouvant être représentée de façon réaliste sur une scène de théâtre, celle-ci est signifiée par la présence de l'interviewer dans la salle, vers le haut des gradins. L'immersion physique des spectateurs dans le lieu fictionnel de Baikonour accentue l'effet de ludique de la scène.

L'analyse de ces scènes permet de comprendre comment Candel fait du jeu l'objet même de sa représentation, comment elle en fait « apparaitre la Forme afin de la transformer en moyen d'expression ${ }^{25} \gg$. La fonctionnalité des signes est privilégiée au détriment du réalisme ou de l'illusionnisme.

\section{Plongée dans le théâtre}

Décrit selon l'angle des références et des enchâssements d'images, le dispositif théâtral ne se résume pourtant pas seulement à montrer des images baroques par la forme. Il en déplie également de façon ludique le sens latent qui réside dans « cette question, ironique et scandaleuse à la fois, des origines $^{26} \gg$.

\section{Theatrum mundi/Mundum theatri}

La quête du Léviathan obsède le documentariste Serge, l'invité malheureux de Michel. La représentation picturale de la créature mythique, plaquée sur d'immenses panneaux de bois, viendra finalement habiller le fond de scène. La solitude et l'ennui s'immiscent dans les scènes d'apesanteur des astronautes confrontés à l'immensité de l'univers et de ses secrets. Cette exploration spatiale se réalise de manière littérale sur scène, sous la forme d'une exploration de l'espace-temps scénique. Le décalage entre les corps et les voix, par exemple, rend sensible la mélancolie et le sentiment d'anomie de ces hommes déracinés. De même, La Leçon de musique applique de manière littérale (et théâtrale) les principes de la peinture flamande sur scène, c'està-dire de dépeindre avec précision une scène de genre, et, par l'ekphrasis, de confondre la description et l'exégèse de la scène $e^{27}$.

Parallèlement, l'optique dialectique ironique se retourne aussi contre le théâtre comme outil didactique. Ainsi, la virtuosité du solo de danse de Dominique Mercy dans Nelken de Pina Bausch est-elle remplacée par un jeu sur l'absence de virtuosité. La même scène est reproduite, mais comme évidée de son fondement. Dans la création originale, Dominique Mercy interpelait le public de ballet sur son goût pour la perfection technique, qui le pousse à applaudir plutôt qu'à ressentir. Ses pirouettes étaient accompagnées d'interpellations au public comme «Ok do you want to see something » et «What else do you want to see? » et la scène s'étirait de façon impressionnante 
dans le temps, avec provocation. Dans Le Goût du faux, ces interpellations accusatrices sont remplacées par le comique des « C'est ça que vous voulez ? » que lance une actrice à moitié nue. Le traitement du costume et de la chorégraphie grossissent le trait de la caricature : la danseuse est simplement vêtue d'une culotte en coton, et non du tutu que Mercy lui-même portait ; ses jetés et fouettés sont peu convaincants. Ce choix de mise en scène plein d'humour montre l'importance du rôle du corps et du jeu dans la production du caractère ludique au théâtre, au-delà de tout artifice.

\section{L'ekphrasis au service de la scène}

Dans ce maillage de saynètes, l'ekphrasis apparait comme l'un des procédés privilégiés pour réinjecter du ludisme dans la représentation. Présente à plusieurs moments dans la représentation, il convient ainsi de définir l'ekphrasis avant d'en analyser plus en détail le fonctionnement dans Le Goût du faux.

Historiquement, l'ekphrasis est d'abord une figure de rhétorique dont le principe est de « faire voir par les mots ». On la retrouve dans des récits comme chez Philostrate ou dans le chant XVIII de l'Iliade où Homère décrit le bouclier d'Achille - où elle prend en charge la description d'images par le langage. L'ekphrasis a pour vocation d'épuiser par les mots l'objet décrit, proposant donc une description minutieuse, mais en ménageant toujours un espace à la réception, c'est-à-dire à la fonction phatique du langage. Son utilisation ne se limite pas au récit : au théâtre, l'ekphrasis est utilisée dans la tragédie romaine où, selon Pierre Katuszewski, elle permet de produire sur scène des images impossibles à montrer par la simple incarnation physique. La parole des acteurs, qui vient se superposer à leurs corps, crée des images qui seraient autrement impossibles à représenter, comme c'est le cas pour les fantômes. De fait, l'ekphrasis ménage des espaces heuristiques dans le jeu : « Ces projections d'images scéniques que permettent ces récits, bien loin de participer d'une construction représentationnelle de l'extra-théâtralité, participent d'une mise en suspens du sens et des sens de l'œuvre pour mieux donner à voir les techniques et les procédés de la machine à produire du spectacle $^{28}$. $\gg$ L'un des effets premiers de l'ekphrasis est par conséquent de produire un type d'expérience et de plaisir particuliers.

Dans Le Goût du faux, l'ekphrasis fonctionne également sur ce principe de suspension du sens et de la vue au bénéfice d'un dévoilement des procédés du théâtre. Mais, là où ce procédé était mis au service d'une émotion tragique chez Sénèque, l'ekphrasis est mise au service du rire et de l'ironie. En effet, lorsqu' ils arrivent sur scène, les quatre personnages sont placés autour du piano : le claveciniste assis sur le tabouret du piano, le violoncelliste à sa 
droite, la chanteuse en face de lui et au fond en retrait, à la limite des coulisses, «l'homme mystérieux $\gg$.

Claveciniste - Il y a un homme en bordure du tableau, qui observe la scène, comme un intrus, et on dirait qu'il rentre dans la scène.

Chanteuse - En fait, il pourrait tout aussi bien être en train de sortir.

Claveciniste - Ouais, il rentre ou il sort, en tout cas, il cherche sa place ${ }^{29}$.

Jouant à représenter l'image qui est en train d'être commentée, chaque personnage décrit pour les spectateurs la composition à laquelle il participe physiquement. Et alors que chacun apporte sa vision personnelle de l'image, une dichotomie s'installe entre ce qui est dit et ce qui est représenté par le groupe. Contrairement à son fonctionnement en littérature, cette ekphrasis crée du trouble, des versions rivales qui coexistent mais ne forment ni une image vraisemblable, ni une composition traduisible en peinture. Le sens symbolique de l'image est d'abord discuté : le claveciniste lui donne une origine mystique, liée à un prétendu culte des « Côtistes »- des peintres flamands qui se retrouvaient dans les cimetières pour se prêter à d'obscures pratiques - tandis que d'autres personnages lui opposent une version dans laquelle «l'homme mystérieux » n'est pas le point de fuite du tableau, mais le peintre lui-même. Comme pour donner du crédit à cette alternative, un personnage figurant le peintre, en habit de velours, entre en fond de scène. L'ekphrasis se révèle être une sorte d'hypotypose performative.

Ce principe performatif de la parole dans l'ekphrasis produit finalement la conclusion de la scène. Ainsi, la chanteuse interrompt-elle le claveciniste en lui criant : « Vous avez un gros insecte sur l'épaule !». Cette phrase marque le retour dans la fiction picturale et induit un changement de niveau dans la représentation, du discursif au dramatique, créant une sorte de rivalité entre ces deux niveaux. Les alternatives jouées irréalisent ce qui a été montré physiquement sur scène dans l'image première. Le jeu dramatique transforme l'image statique, incarnée, en une virtualité réalisée, pourtant vue et visible. Elle déconstruit l'image scénique et la remplace par une autre image mentale que le spectateur doit actualiser par son imagination.

Élevée au rang de procédé heuristique du Goût du faux, l'ekphrasis s'adapte à chaque référence artistique, selon sa nature : la peinture hollandaise produit une ekphrasis descriptive, tandis que la peinture du Quattrocento, une ekphrasis narrative. Elle épouse et révèle à la fois le mode de fonctionnement des références utilisées. Dans la première, la description des attitudes et des places des personnages produit un jeu métathéâtral, assumé comme tel, tandis que 
dans le second cas, l'identité entre la comédienne et le « personnage-palimpseste » est recherchée à travers l'hyper-investissement de la parole et de la voix, ce qui produit une image statique, renforcée par le désinvestissement du corps.

\section{Conclusion : des effets du faux au théâtre}

Le Goût du faux apparaît donc comme un dispositif immersif cognitif, ou pour le dire autrement, un dispositif pulsionnel : la pièce stimule la connaissance et la reconnaissance, elle étire même le $\ll$ rôle du spectateur ${ }^{30} \gg$ d'une activité cognitive à une activité « pulsionnelle » avec l'ekphrasis. En tant que telle, l'expérience théâtrale encourage un autre type de connaissance du monde, ni philosophique, ni intellectuelle mais pathétique et spectaculaire. Le théâtre produit les possibilités d'une réception, voire d'une connaissance « qui ne passe pas par l'intelligence $[\ldots]$ mais par l'émotion ${ }^{31} \gg$, pourrait-on dire après Klossowski, une connaissance pathétique. Et le pathétique ne se réduit pas aux pleurs seulement. Le pathétique, c'est rire, pleurer et honnir une gamme d'émotions bien plus large et complexe que la simple catharsis de la tragédie, fonctionnant à force de terreur et pitié.

En effet, le dispositif scénique tout entier est construit comme un espace encourageant l'expérience pathétique. Le revêtement plastique recouvrant le plateau, les murs peints et les rideaux de scène, tous noirs, fonctionnent comme les « planches noires de l'Atlas » d'Aby Warburg où l'historien de l'art échantillonne le chaos «par images interposées ${ }^{32}$ ». La diversité des scènes analysées suit en effet le même principe de variété des représentations que celui de l'Atlas Mnémosyne où les astra et les monstra cohabitent et donnent du sens au chaos du monde et dans lequel l'émotion, le pathos, est un principe organisationnel préféré au logos.

L'omniprésence des références culturelles qui modèlent littéralement la pièce est également, pour Jeanne Candel, une manière de mettre l'emphase sur la relation que le spectateur entretient avec le réel : la connaissance du monde est toujours médiée par des images, et cette hyper-médiation est symptomatique d'un rapport au monde « hyperréalisés ${ }^{33} \gg$. Cependant, l'ekphrasis théâtrale apparaît comme un moyen performatif de faire de la représentation théâtrale le lieu de dépassement du sentiment d'anomie produit par l'hyperréalisation du réel. Car la mise en scène du Goût $d u$ faux ne propose pas de démonstration théorique, mais une expérience esthétique qui réintroduit du ludique et de l'imaginaire là où une suspicion de passivité et de crédulité planaient. Ainsi, le dispositif théâtral baroquisant de Candel encourage-t-il le 
spectateur à jouer son rôle d'interprète sensible des images scéniques, et c'est lorsqu'il s'exécute qu'il éprouve enfin du plaisir.

\section{Notes}

1. Propos recueillis par Stéphane Bouquet pour le Festival d'Automne à Paris - reproduit dans le programme de salle du spectacle au Théâtre de la Cité Internationale.

2. La bande-annonce du spectacle, accessible sur la plateforme de vidéo YouTube, permet de se rendre compte de l'esthétique générale de la pièce. Pour la définition du postdramatique au théâtre, nous renverrons à l'essai - tout autant critiqué que référence devenue incontournable - d'Hans-Thies Lehmann, Le Théâtre postdramatique, Paris, L'Arche, 2002.

3. La signification de ce terme, mimèsis, étant sujet à de nombreux débats, nous prendrons comme point de référence les travaux de Florence Dupont, et plus précisément Aristote ou le vampire du théâtre occidental, où la mimèsis est définie comme " représentation » au sens large et applicable à tous les arts. Aristote ou le vampire du théâtre occidental, Paris, Flammarion, coll. « Libelles », 2007.

4. Selon le Trésor de la Langue française, le concept de faux en esthétique renvoie à ce « qui est contraire à la justesse, au naturel ». [consulté le 29 mars 2015]. Disponible sur : http://atilf.atilf.fr/dendien/scripts/tlfiv5/visusel.exe?12;s=2941281690;r=1;nat=;sol=1;

5. Sur la démonstration de la sacralisation de la fable, ou muthos aristotélicien, nous renvoyons également à l'analyse par Florence Dupont du glissement de la tragédie grecque antique comme pratique rituelle et performative à un seul texte poétique - régi par le muthos. Florence Dupont, Aristote ou le vampire du théâtre occidental, op. cit.

6. Cf. André Helbo et al., "Théâtre et spectacle vivant. Mutations contemporaines ", Revue internationale de philosophie, 2011/1, n 255, p. 85-101.

7. Sur cette question de changement de paradigme dans la création contemporaine, de la question de la distance critique à la connaissance empathique, cf. Catherine Grenier, $L a$ Revanche des émotions. Essai sur l'art contemporain, Paris, Seuil, coll. " Fiction et Cie ", 2008.

8. Denis Guénoun, L'Exbibition des mots. Une idée politique du théâtre, La Tour d'Aigues, Éditions de l'Aube, coll. « Monde en cours», 1992, p. 52.

9. Nous empruntons le concept de «pli » à Deleuze. Dans Le Pli. Leibniz et le Baroque, le concept de pli est lié autant au caractère citationnel du Baroque, qui ne crée a priori rien mais qui emprunte aux arts et cultures passés, qu'à sa nature qui lie la forme et la pensée, les parties hétérogènes et le tout. Gilles Deleuze, Le Pli. Leibniz et le Baroque, Paris, Minuit, 1988.

10. Nous employons «baroquisant » et non «baroque » pour décrire l'esthétique de Jeanne Candel car il s'agit plus d'une source d'inspiration de l'époque baroque que d'une reconstitution fidèle de ses préceptes.

11. Svetlana Alpers, L'Art de dépeindre, Paris, Gallimard, coll. «Bibliothèque illustrée des histoires », 1990. 
12. Agnès Jaoui et Jean-Pierre Bacri, Cuisine et dépendances. La pièce a été montée pour la première fois au Théâtre La Bruyère à Paris en 1991. Agnès Jaoui et Jean-Pierre Bacri, Le Goût des autres, 2000.

13. Dans L'Empire ludique, Aurélien Fouillet resitue la résillusion dans son contexte historique, en tant qu'elle est héritière de l'enromancement médiéval et du bovarysme du $\mathrm{XIX}^{\mathrm{e}}$ siècle. Selon lui, « le milieu dans lequel se déploient les fictions identitaires est l'espace de la résillusion, c'est-à-dire un espace profondément poreux et dans lequel intériorité, environnement, imagination et communautés se rejoignent ", Aurélien Fouillet, L'Empire ludique, Lormont et Paris, Éditions François Bourin, coll. « Penser Le Monde/Société », 2014, p. 105.

14. La « scène ambulatoire » est le nom donné par Sarrasin dans son Discours de la tragédie (1639) à un « dispositif qui donne à voir l'ensemble des différents lieux de l'action, dès le début de la représentation, et sans changements de décor », et dans lequel « chaque compartiment figure l'un des lieux de l'action, par allusion, par raccourci, et non pas de façon descriptive ", in L'Automne de l'imagination, Splendeurs et misères de la représentation, $\mathrm{XVI}^{\mathrm{e}-\mathrm{XXI}}{ }^{\mathrm{e}}$ siècle, Bern, Peter Lang, 2012, p. 138 et p. 137.

15. Dans le même entretien de Stéphane Boitel avec Jeanne Candel, présenté dans le dossier de presse du Goût du faux au Théâtre de la Cité internationale en 2015, le terme de « low cost» est utilisé pour décrire l'esthétique de la scène. Ce terme nous semble insatisfaisant dans la mesure où il confond les coûts de production et la nature de la réalisation esthétique.

16. En effet, selon Roland Barthes, le costume de théâtre doit toujours garder sa valeur de fonction, au-delà des tentations de "vérisme historique ", de surenchère " esthétique " sans lien avec la pièce, ou " d'hypertrophie de la somptuosité ». Et d'ajouter : "Le costume doit toujours garder sa valeur de pure fonction, il ne doit ni étouffer ni gonfler la pièce, il doit se garder de substituer à la signification de l'acte théâtral, des valeurs indépendantes », Roland Barthes, «Les Maladies du costume de théâtre » (1955) dans Écrits sur le théâtre, Paris, Points, coll. «Essais », 2015, p. 137-146.

17. Lionel Abel, Metatheatre. A New View of Dramatic Form, New York, Hill and Wang, 2002. Le terme métathéâtre ou metatheatre, ainsi qu'un premier essai de définition, sont donnés dans l'article « Metatheatre: Shakespeare and Calderón », p. 59-72.

18. Aurélien Fouillet, op. cit.

19. André Helbro et al., "Théâtre et spectacle vivant. Mutations contemporaines ", p. 94. Au théâtre, l'immersion cognitive, et parfois physique, du spectateur nécessite à la fois son identification aux personnages, et sa prise de distance par rapport à l'environnement physique immédiat. Or l'homomatérialité du théâtre est ce qui permet et freine à la fois l'immersion : le personnage est un être humain, la plupart du temps, comme le spectateur, mais le lieu et le temps de la représentation affaiblissent ce sentiment d'identification car le spectateur sait être en présence d'un simulacre au théâtre.

20. La théâtralité étant une notion polysémique et complexe, nous nous référons ici aux travaux de Josette Féral pour qui la théâtralité a à voir avec l'acte de connaissance et de création du spectateur : "La théâtralité serait ainsi le résultat d'une action qui consisterait pour le sujet, confronté à des actions, des événements du réel ou des moments scéniques, à opérer dans le réel soumis à son regard une série de clivages spatiaux et temporels par lesquels il créerait "un lieu de fiction" dans lequel le jeu peut advenir ", 
Josette Féral, «Les Paradoxes de la théâtralité », Théâtre/Public, « Entre deux. Du théâtral et du performatif ", n 205, juillet-septembre 2012, p. 10.

21. Pablo Picasso, cité par Françoise Gillot, Vivre avec Picasso, Paris, Éditions CalmannLévy, 1991, p. 293.

22. Dans un article intitulé "Le rôle de la musique dans un film ", cette dernière est jugée réussie quand le spectateur oublie sa présence : «le meilleur témoignage qu'on puisse rendre de la musique est le suivant : si on ne doit pas remarquer sa présence, on doit déplorer son absence », [S. a.], Séquences : la revue de cinéma, n 14, septembre 1958, p. 11.

23. Jean-Luc Guionnet, «Scène \& musique : représentation \& stratégies », Théâtre/Public, "Le Son du Théâtre II : Dire l'acoustique », n 199, mars 2011, p. 47.

24. Walter Benjamin, «Le Surréalisme - le dernier instantané de l'intelligentsia européenne », Euvres 2, Paris, Gallimard, coll. « Folio/Essais », 2000, p. 131.

25. Gilles Deleuze, op. cit., p. 49.

26. Jeanne Candel, "Interview : Jeanne Candel, "Le Goût du faux et autres chansons", festival d'automne», in Smarande Olcèse, Inferno. [Enligne].24 novembre 2014 [consulté le 20 février 2015]. Disponible sur : http://inferno-magazine.com/2014/11/24/interview-jeanne-candel-le-gout-du-faux-et-autres-chansons-festival-dautomne/

27. Dans son essai sur la peinture flamande, Svetlana Alpers développe la thèse selon laquelle la dimension descriptive de cette peinture - a contrario du style narratif italien de la même époque - relève d'un souci de compréhension scientifique du monde : « La peinture hollandaise du XVII ${ }^{\mathrm{e}}$ siècle avait émis une hypothèse particulière, à savoir que découverte et création - notre exploration de l'univers et l'action qui nous permet de le modeler - ne font qu'un ", Svetlana Alpers, op. cit., p. 69.

28. «L'ekphrasis : quand les mots font le spectacle », Teresa Orecchia-Havas, Anne Surgers, Marie-José Tramuta, Baptiste Villenave et Julie Wolkenstein (dir.), Le Regard à l'œuvre. Lecteurs de l'image, spectateurs du texte, Caen, mars 2014, p. 36. Disponible sur : http:// www.unicaen.fr/recherche/mrsh/laslar/2669

29. Dialogues extraits de la bande-annonce de la pièce : FESTIVALDAUTOMNE, "Jeanne Candel / Le Goût du faux et autres chansons » [consulté le 31 mai 2015]. Disponible sur : https://www.youtube.com/watch?v=AjgxsUUnEHY.

30. Le rôle du spectateur ", traduction de l'anglais " the share of the spectator " est une expression forgée par E. H. Gombrich dans L'art et l'illusion pour désigner l'ensemble des actes perceptifs et psychologiques par lesquels le spectateur fait exister l'image. S'inscrivant dans la continuité des travaux de Rudolf Arnheim (1969) sur le rapport de l'image au réel, Gombrich démontre qu'aucun regard n'est innocent, que son rôle est « projectif » et que l'image est un phénomène lié à l'imagination du spectateur comme à celle du créateur.

31. Catherine Grenier, op. cit., p. 27.

32. George Didi-Huberman, "Échantillonner le chaos. Aby Warburg et l'atlas photographique de la Grande Guerre ", Études photographiques, n 27 "Images de guerre, photographies mises en page ", 27 mai 2011, [En ligne], mis en ligne le 18 novembre 2011 [page consultée le 20 février 2016]. Disponible sur : http:// etudesphotographiques.revues.org/3173 
Théâtres du geste, du jeu et de la voix

33. Pour Baudrillard, le simulacre et la simulation sont des expériences négatives, dans la mesure où elles ont envahi notre quotidien. Selon lui, la simulation, comme le simulacre, se substituent au réel : "Elle est la génération par les modèles d'un réel sans origine ni réalité : hyperréel ». Jean Baudrillard, Simulacres et simulation, Paris, Éditions Galilée, 1981, p. 10. 